\title{
Coping retained removable partial denture an alternative to fixed partial denture: A case report
}

\author{
Arthi Ambayiram, Ahila Singaravel Chidembaranathan* and Muthukumar Balasubramanium \\ Department of Prosthodontics, SRM Dental College, Ramapuram, Chennai, India
}

\begin{abstract}
Overdenture therapy is a kind of preventive prosthodontics treatment modality that preserves the remaining natural teeth and roots. The periodontal membrane with its proprioceptive mechanism can be preserved by the overdenture therapy. This case report presents a tooth supported removable partial denture which influence esthetics and improved function.
\end{abstract}

\section{Introduction}

Loss of teeth will lead to a rapid reduction in the height of the alveolar process. This morphologic change has been considered as a major disease entity. The presence of teeth maintains the alveolar ridge integrity. The advantages of preserving teeth or roots influence the psychological benefits to the patient, tactile discrimination and improved stability and retention of the denture [1]. Overdenture is any removable dental prosthesis that covers and rests on one or more remaining natural teeth, the roots of natural teeth or dental implants [2]. This case report describes a treatment method of tooth supported removable partial denture.

\section{Case report}

A 56-year-old male patient reported the Department of Prosthodontics at SRM Dental College and Hospital, Ramapuram, Chennai, India with a chief complaint of fractured fixed partial denture in the upper right teeth region and he wants to restore the teeth. On clinical examination, it was found that $11,12,14,16,24$ and 25 are missing in maxillary arch and $36,44,45$ and 46 are missing in mandibular arch. The teeth preparation had been done previously in 13,15 and was periodontally sound. Due to the financial and economic reasons of the patient, tooth supported removable partial denture has been given as a alternative treatment option to fixed partial denture using the existing prepared tooth as abutment.

\section{Treatment procedure}

1. Diagnostic impression has been made with irreversible hydrocolloid impression material and space was assessed for copings.

2. Definitive impression was made with polyvinylsiloxane impression material and cast was poured with die stone.

3. Wax pattern of the copings in relation to 13 and 15 were fabricated. The wax patterns are invested and casting has been done. The fit of the coping was verified in the cast and in the patient's mouth.

4. The copings were cemented with Glass Ionomer type 1 luting cement. The impression was then made with irreversible hydrocolloid impression material and the cast was poured with dental stone.
5. Denture base and occlusal rims were fabricated, and tentative jaw relation was done, followed by articulation and teeth arrangement.

6. For occlusion of the maxillary teeth, additional row of teeth has been placed in the mandibular removable dental prosthesis. The wax trying has been done. For stabilization of lower denture, clasps had been placed in relation to 37 and 47.

7. The denture has been processed. Finishing and polishing of the denture has been done and the denture was inserted. Patient was recalled after 24 hours for postinsertion check-up (Figures 1-4).

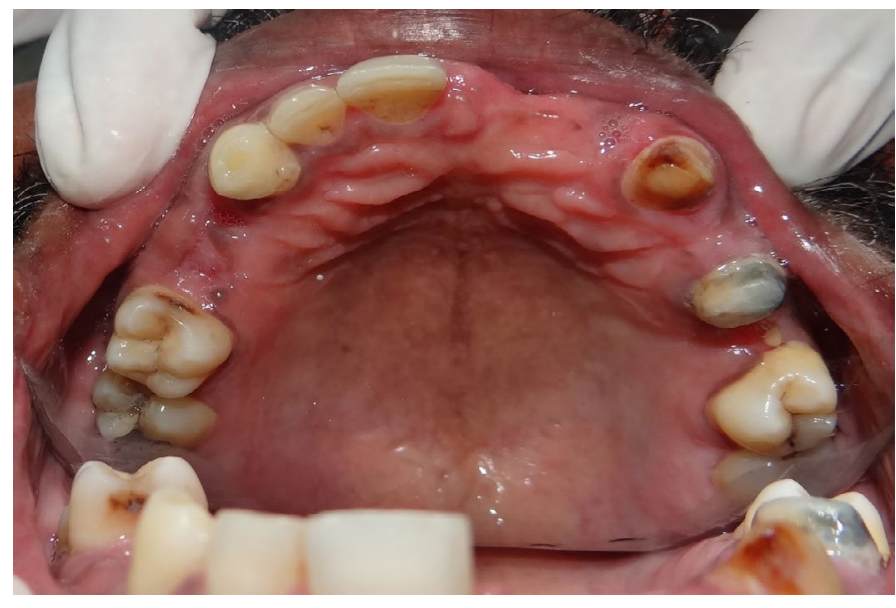

Figure 1. Intra oral view -maxilla

*Correspondence to: S C Ahila, Associate Professor, Department of Prosthodontics, SRM Dental College, Ramapuram, Chennai-89, India, Tel: 9443607653, E-mail: ahilasc@yahoo.co.in

Key words: over denture, removable partial denture, proprioception

Received: March 17, 2019; Accepted: May 01, 2019; Published: May 03, 2019; 


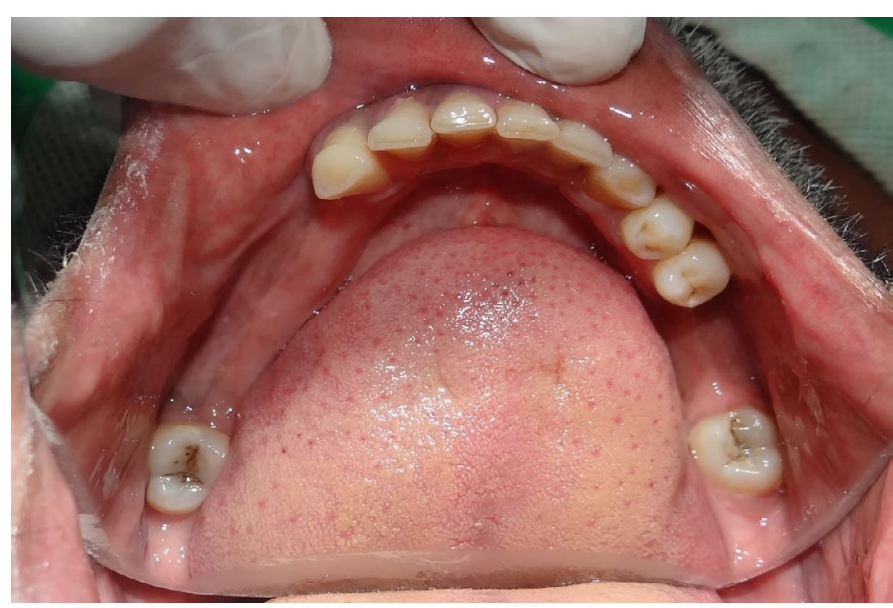

Figure 2. Intra oral view-mandible

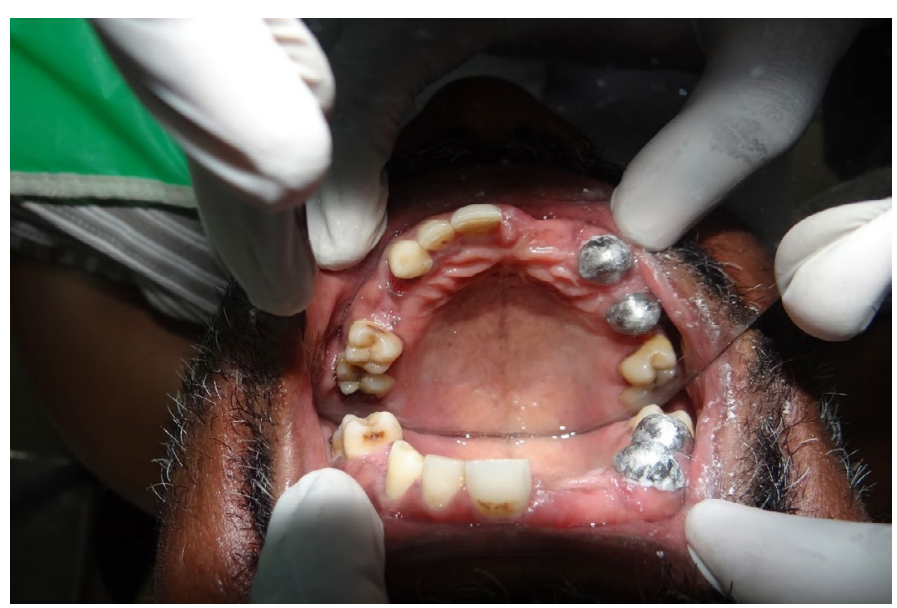

Figure 3. Coping luted over the prepared tooth

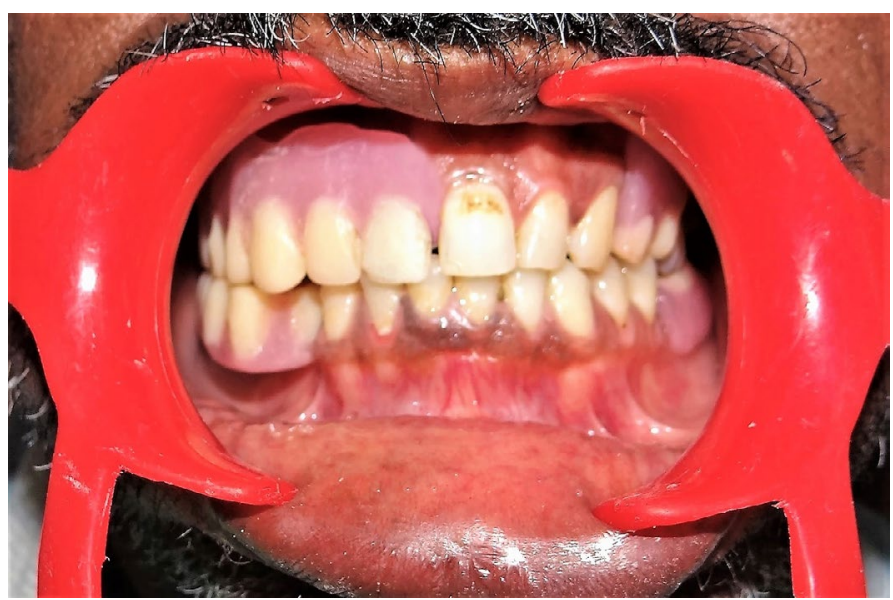

Figure 4. Rehabilitation with removable partial denture

\section{Discussion}

The problem associated with overdenture was periodontal and endodontic failure of retained abutment tooth. The improper coverage of tooth with coping results in open margins that leads to caries and poor oral hygiene [3]. Paul A. Miller stated that copings or castings had to cover the prepared abutment tooth. All exposed surfaces are covered to prevent any carious lesion developing in future [4].

Vishakha Bhandare reported that the selection of copings depends on angulations of the root to the occlusal plane, amount and quality of bone support, abutment location and chewing pattern. The use of copings improves the retention and the stability of the overdenture with proper maintenance program [3].

\section{Conclusion}

Retaining teeth improves patient satisfaction and improves the health of the residual ridge. Copings retained removable partial denture can be used as option, which increases the retention of the denture.

\section{References}

1. Preiskel H (1996) Overdentures made easy. A guide to implant and root supported prostheses.

2. The Academy of Prosthodontics (2017) The glossary of prosthodontic terms. J Prosthet Dent 117: 1-105.

3. Bhandare V (2015) Prosthodontic rehabilitation with overdenture using modified impression technique: A case report. IOSR J Dent Med Sci 14: 2279-2281.

4. Miller PA (1958) Complete dentures supported by natural teeth. J Prosthet Dent 8: 924-928.

Copyright: $@ 2019$ Ambayiram A. This is an open-access article distributed under the terms of the Creative Commons Attribution License, which permits unrestricted use, distribution, and reproduction in any medium, provided the original author and source are credited. 